COMBUSTION

HARDWOOD

FURAN

ETHENYLFURANS

ANALYSIS
GLOWING

$\mathrm{BIRCH}$

2-METHYLFURAN

DIMETHYLFURANS

GC - MS

Open access accepted manuscript version of

Journal of Chromatography A 753 (1996) 151-155

doi:10.1016/S0021-9673(96)00534-1

\title{
Gas chromatographic separation of volatile furans from birchwood smoke
}

Gunnar Barrefors, Susan Björkqvist, Olle Ramnäs and Göran Petersson

A related analytical study was made of the wide range of volatile hydrocarbons from wood burning 


\title{
Gas Chromatographic Separation of Volatile Furans from Birchwood Smoke
}

\author{
Gunnar Barrefors, Susan Björkqvist, Olle Ramnäs and Göran Petersson \\ Department of Chemical Environmental Science \\ Chalmers University of Technology \\ 41296 Göteborg, Sweden
}

\begin{abstract}
Furan, 2-methylfuran, 3-methylfuran, 2,5-dimethylfuran and the five isomeric $\mathrm{C}_{6}$ alkylfurans, two $\mathrm{C}_{7}$ alkylfurans, and three $\mathrm{C}_{6}-\mathrm{C}_{7}$ alkenylfurans were determined in birchwood smoke by adsorbent sampling, gas chromatography and mass spectrometric techniques. Retention data, mass spectral data, and quantitative proportions are given. Furan and 2-methylfuran are major components. Contrary to other oxygen-containing compounds, the furans elute from the aluminium oxide column widely used for volatile $\mathrm{C}_{2}-\mathrm{C}_{8}$ hydrocarbons. The furans are formed mainly from glowing and smouldering combustion.
\end{abstract}




\section{Introduction}

Furan and 2-methylfuran appear as prominent peaks in the chromatograms when $\mathrm{C}_{2}$ $\mathrm{C}_{8}$ volatile hydrocarbons in biomass smoke samples are analyzed on aluminium oxide columns $[1,2]$. This indicates that furan and alkylfurans might be selectively analyzed on these porous-layer open tubular (PLOT) columns, since other oxygen-containing compounds are normally not eluted. A wide range of furan-related carbonyl and other polar compounds are formed in large amounts from pyrolysis of wood polysaccharides $[3,4,5]$. Alkylfurans and alkenylfurans have been identified by GLC-MS techniques mainly from other sources such as roasting aroma [6]. Cereal molds form 3-methylfuran [7], and specific furans may also be of interest in sick buildings [8].

The purpose of this study was to characterise volatile furans in birchwood smoke which may be of interest with respect to human exposure and as indoor and outdoor wood-smoke tracers in studies of air pollutants. A further aim was the evaluation of aluminium oxide columns and GLC-MS techniques for the assessment of furans.

\section{Experimental}

\subsection{Wood burning}

Dry chips and pieces of Scandinavian birchwood were burnt in a ceramic pot (1 1). A larger clay pot, with a sampling hole in the bottom, was placed upside down over the ceramic pot for the purpose of collecting smoke from choked fire and from glowing birchwood. Smoke from flaming fire was collected by keeping the clay pot as high above the fire as needed to prevent choking.

Complementary burning experiments were performed in a wood-stove with chimney sampling [2]. 


\subsection{Sampling and gas chromatography}

The methods for sampling and gas chromatography were essentially those described in some detail in a previous study of volatile hydrocarbons from biomass burning [1]. Smoke samples in the range $10-100 \mathrm{ml}$ were taken on triple-layer adsorbent cartridges (Tenax $\mathrm{TA}+$ Carbotrap + Carbosieve S-III). Following thermal desorption $\left(235^{\circ} \mathrm{C}, 14 \mathrm{~min}, 20-\right.$ $40 \mathrm{ml} \mathrm{min}-1 \mathrm{He}$ ) into a cold trap ( $5 \mathrm{~m} \mathrm{x} 0.32 \mathrm{~mm}$ I.D. empty fused silica capillary in liquid $\mathrm{N}_{2}$ ), gas chromatographic separation was performed on an aluminium oxide column $\left(\mathrm{Al}_{2} \mathrm{O}_{3} / 5 \% \mathrm{KCl}\right.$, PLOT, $50 \mathrm{~m} \times 0.32 \mathrm{~mm}$ I.D. fused silica, Chrompack). The carrier gas was $\mathrm{He}\left(30 \mathrm{~cm} \mathrm{~s}^{-1}\right)$, and the final oven temperature $200^{\circ} \mathrm{C}$ (preceeded by $110-200^{\circ} \mathrm{C}, 4^{\circ} \mathrm{C}$ $\left.\min ^{-1}\right)$. Alternatively, gas syringe samples were taken for gas injection via a $3.2 \mathrm{ml}$ injection coil.

The flame ionisation response was determined from gravimetrically prepared liquid mixtures of reference compounds. Gaseous references in the ppm range were obtained by injecting a weighted amount of the liquid into a glass vessel (50 1). The flame ionisation response was found to be similar for the available reference furans, and $60 \%$ of the response of hydrocarbons was used for the quantitative evaluations.

Separations with mass spectrometric detection were performed on a methylsilicone column (50 m $\times 0.32 \mathrm{~mm}$ I.D., fused silica WCOT, $1 \mu \mathrm{m}$ DB-1, J\&W). Gas samples $(0.5-1 \mathrm{ml})$ of high-concentration birchwood smoke were taken and injected with a $1 \mathrm{ml}$ gas-tight syringe. Linear temperature programming $\left(3^{\circ} \mathrm{C} \min ^{-1}\right.$ from $\left.-30^{\circ} \mathrm{C}\right)$ was applied, and the retention of the furans was determined relative to co-separated reference alkanes.

Carbon dioxide was determined separately as previously described [2] by gas chromatography on a porous polymer column (HayeSep Q, $80^{\circ} \mathrm{C}$, gas-valve injection) with thermal conductivity detection. 


\subsection{Mass spectrometry}

The mass spectrometer was a Varian Saturn II ion trap GC-MS instrument used with electron impact ionization. The ion trap temperature was $120^{\circ} \mathrm{C}$. One mass spectrum $(m / z 35-200)$ per second was recorded. Computer storing and dedicated data programs permitted straightforward reconstruction of ion chromatograms and of integrated background-corrected mass spectra of separated compounds. The total ion current response $(m / z 35-200)$ of two $\mathrm{C}_{6}$ reference furans was found to be about $80 \%$ of the response of heptane. An observed $20 \%$ increase in response with each additional carbon atom was also considered when calculating furan proportions from total ion chromatograms.

Ion chromatograms and mass spectra were used for the identification of furans in the matrix of a wide range of different compounds in birchwood smoke. The identities of furan, 2-methylfuran, 2-ethylfuran and 2,5-dimethylfuran were confirmed by comparisons with authentic reference furans (Aldrich, 97-99\%). Data-stored reference NIST spectra were available also for 3-methylfuran, 2,4-dimethylfuran and 2-ethyl-5methylfuran. Published mass spectral data [6] are available for all these and several additional furans, including 2,3-dimethylfuran, 2,3,5-trimethylfuran and the three reported alkenylfurans. Structural assignments for all reported $\mathrm{C}_{6}$ and $\mathrm{C}_{7}$ furans were based on comparisons of mass spectra, interpretations of mass spectrometric fragmentations, relationships between structure and retention, and relationships between structure and quantitative proportions, as discussed below. 


\section{Results and discussion}

\subsection{Chromatographic separations}

The chromatogram in Fig. 1 illustrates the separation on an aluminium oxide column of furans and related unsaturated hydrocarbons from choked birchwood burning. The separation and proportions of the $\mathrm{C}_{2}-\mathrm{C}_{8}$ hydrocarbons from the same pot-burning experiment were reported in a previous study [1], permitting extended comparisons. The $\mathrm{C}_{2}-\mathrm{C}_{5}$ hydrocarbons are best separated by temperature-programmed analysis below $200^{\circ} \mathrm{C}[1]$, whereas the furans are eluted during continued isothermal analysis at $200^{\circ} \mathrm{C}$.

Furan elutes after isoprene and cyclopentadiene in the same region as minor pentadienes and branched hexenes. The most prominent alkylfuran, 2-methylfuran, appears before benzene. The stronger retention of the 3-methylfuran isomer is explained by a lower steric hindrance from the methyl group to polar interactions between the furan oxygen bridge and the stationary phase. The major $\mathrm{C}_{6}$ furan is 2,5-dimethylfuran which is eluted first of all $\mathrm{C}_{6}$ furans, due to the two methyl groups enclosing the ring oxygen. Several minor $\mathrm{C}_{6}$ and $\mathrm{C}_{7}$ furans appear in the chromatographic range before and after methylbenzene.

The response factors of furan, 2-methylfuran, 2-ethylfuran and 2,5-dimethylfuran relative to reference hydrocarbons were found to be the same for the $\mathrm{Al}_{2} \mathrm{O}_{3}$ column as for a silicone GLC column, demonstrating that furans are quantitatively eluted from the $\mathrm{Al}_{2} \mathrm{O}_{3}$ column. The $\mathrm{Al}_{2} \mathrm{O}_{3}$ column therefore permits a selective determination of furans, free from interferences with other oxygenated compounds which are not eluted from the column. No artifacts from these retained oxygenated combustion products or from the adsorbent cartridges were observed in the chromatograms. The simultaneous determination of volatile $\mathrm{C}_{2}-\mathrm{C}_{8}$ hydrocarbons [1] and volatile furans on the $\mathrm{Al}_{2} \mathrm{O}_{3}$ column may be advantageous in many applications. 


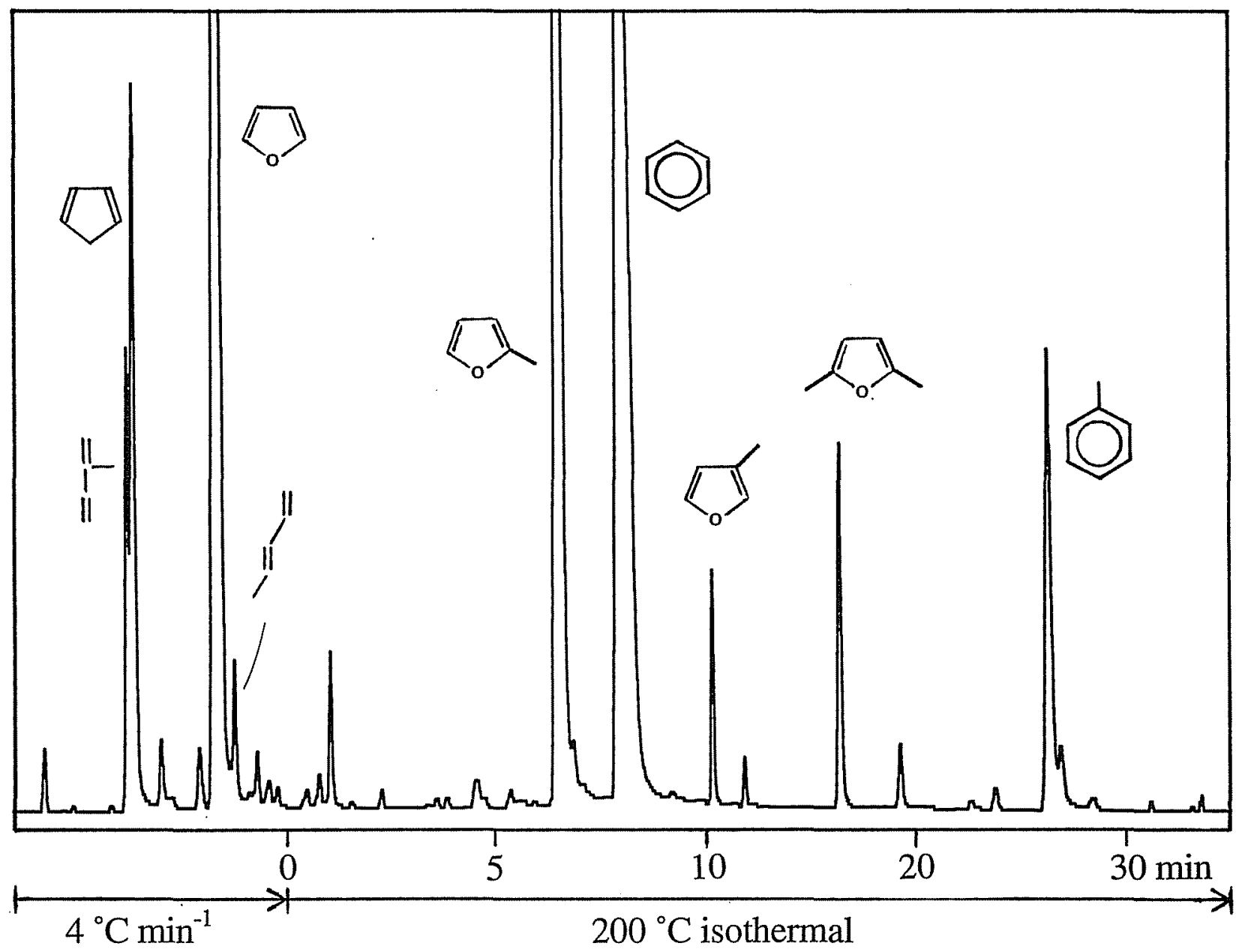

Fig. 1 Gas chromatographic separation on an aluminium oxide column of prominent furans, alkadienes and arenes from birchwood smoke. 
A more comprehensive study of furans in wood smoke was made using a methylsilicone non-polar capillary column linked to a mass spectrometer. In the first column of Table 1, the retentions of the identified furans are given relative to reference alkanes for linearly temperature-programmed analysis. Furan appears near to pentane (500), the two $\mathrm{C}_{5}$ furans near to hexane (600), the eight $\mathrm{C}_{6}$ furans near to heptane (700) and the $\mathrm{C}_{7}$ furans near to octane (800). Isomers substituted adjacent to the furan oxygen at $\mathrm{C}-2$ (and C-5) are eluted before those substituted at $\mathrm{C}-3$ (and $\mathrm{C}-4$ ). On the polar $\mathrm{Al}_{2} \mathrm{O}_{3}$ column, the furans are more strongly retained relative to hydrocarbons, as demonstrated by the elution of furan after hexane and of 2-methylfuran near to heptane. Retention data on other polar stationary phases have been reported for many furan-related compounds $[4,6]$.

\subsection{Ion chromatograms and mass spectra}

The separation of all the $\mathrm{C}_{6}$ furans on the methylsilicone column is illustrated in Fig. 2 by a data-reconstructed mass spectrometric ion chromatogram corresponding to the sum of the molecular ions with $m / z 96$ (alkylfurans) and $m / z 94$ (alkenylfurans). This detection method permits a clear-cut recording of the furans without interferences from other combustion-formed compounds in the same chromatographic region. The presence of lateeluting phenols in large amounts indicated that significant sampling losses of the volatile furans by condensation on particles could be precluded. The high abundance of the molecular ions of the furans renders the detection by these ions very sensitive. The highest response is obtained for the ethenylfurans and the lowest for the ethylfurans which form favoured fragment ions.

The selected ions listed in the second column of Table 1 are of particular value for the mass spectrometric identification of the furans. The aromatic character of the furan ring stabilises the molecular ion and prevents cleavage of the furan ring and of links between 
Table 1

Relative GC retentions, mass spectral data, and quantitative proportions ${ }^{\mathrm{a}}$ of furans in birchwood smoke.

\begin{tabular}{|c|c|c|c|}
\hline & 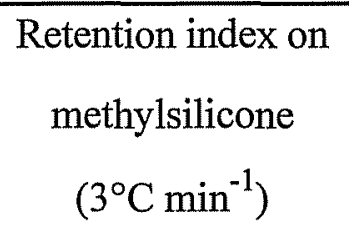 & $\begin{array}{c}\text { Masses }(\mathrm{m} / \mathrm{z}) \text { of } \\
\text { molecular ion and } \\
\text { specific fragment ions }\end{array}$ & $\begin{array}{l}\text { Proportions from } \\
\text { choked fire } \\
\text { (furan } 1.0 \text { ) }\end{array}$ \\
\hline Furan & 492 & 68,39 & 1.0 \\
\hline 2-Methylfuran & 594 & $82,81,53$ & 1.0 \\
\hline 3-Methylfuran & 603 & $82,81,53$ & 0.1 \\
\hline 2,5-Dimethylfuran & 696 & 96,95 & 0.2 \\
\hline 2-Ethylfuran & 690 & 96,81 & 0.04 \\
\hline 3-Ethylfuran & 701 & 96,81 & 0.01 \\
\hline 2,4-Dimethylfuran & 703 & $96,95,67$ & 0.03 \\
\hline 2,3-Dimethylfuran & 705 & $96,95,67$ & 0.03 \\
\hline 2-Ethenylfuran & 710 & $94,66,65$ & 0.04 \\
\hline 3-Ethenylfuran & 713 & $94,66,65$ & 0.01 \\
\hline 3,4-Dimethylfuran & 724 & $96,95,67$ & 0.01 \\
\hline 2-Ethyl-5-methylfuran & 789 & 110,95 & 0.02 \\
\hline 2,3,5-Trimethylfuran & 803 & 110,109 & 0.01 \\
\hline $\begin{array}{l}\text { 2-Ethenyl-5- } \\
\text { methylfuran }\end{array}$ & 815 & 108,107 & 0.02 \\
\hline Benzene & 648 & 78 & 0.5 \\
\hline
\end{tabular}

${ }^{a}$ Calculated from gas chromatograms from the aluminium oxide column (prominent furans and benzene), and from mass spectrometric total ion chromatograms (minor alkylfurans). 


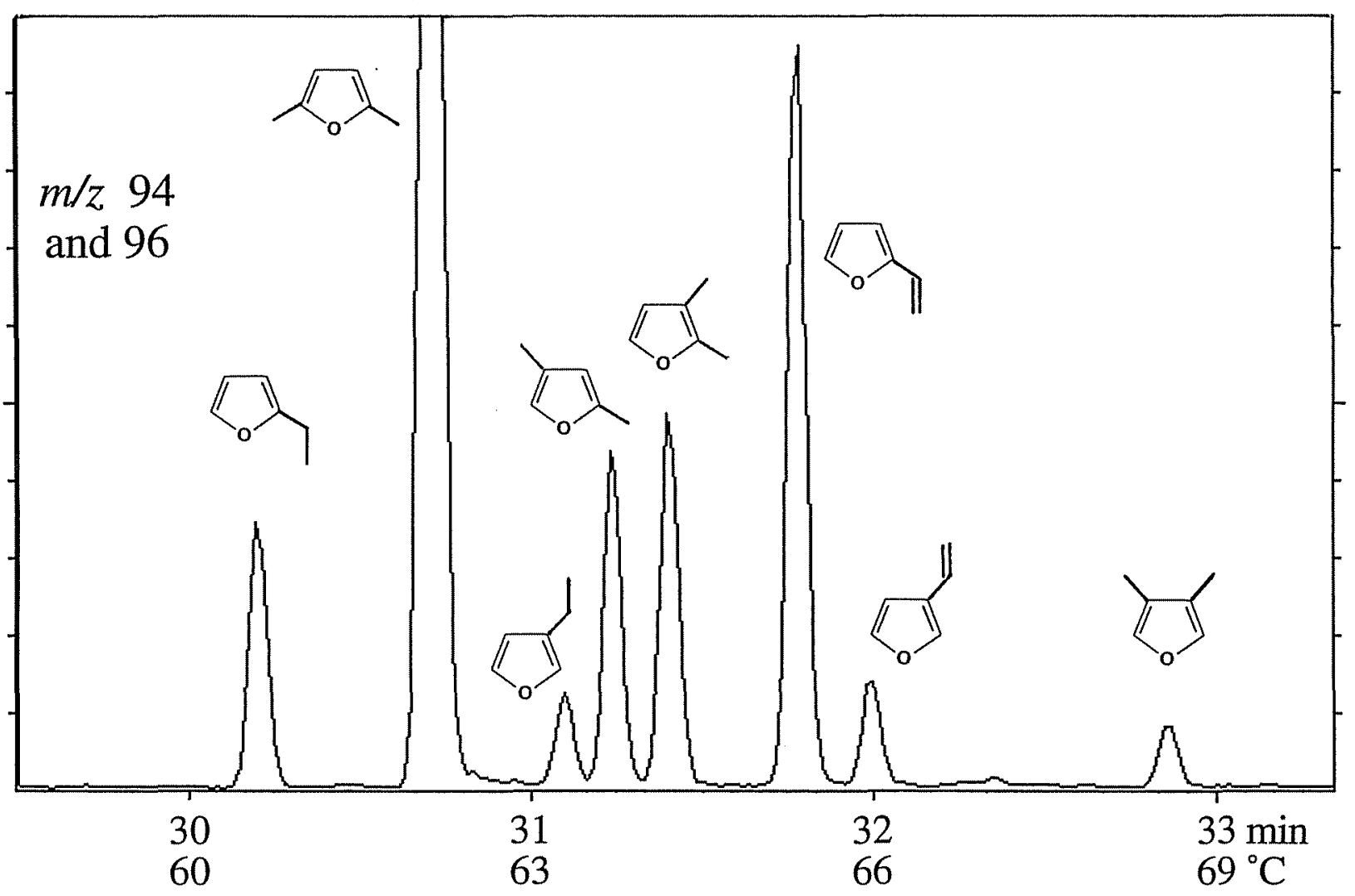

Fig. 2 Mass spectrometric ion chromatogram for the molecular ions of $\mathrm{C}_{6}$ furans as separated on a methylsilicone column. 
the ring atoms and adjacent atoms. The ethylfurans give rise to very abundant $M-15$ ions from cleavage of the ethyl group with formation of a resonance-stabilised ion. The methylfurans give rise to corresponding characteristic $M-1$ ions from loss of a methyl hydrogen atom. These fragmentations are analogous to the well-known $\beta$-cleavage of alkylbenzenes [6]. The mass spectra indicate that several primary fragment ions tend to decompose further by loss of carbon monoxide, corresponding to 28 mass units. The recorded spectra compared well with tabulated spectra published for several of the furans $[6]$.

\subsection{Proportions of furans in wood smoke}

In Table 2, representative quantitative proportions are given for gentle glowing combustion and for flaming combustion in the pot-burning experiments. Differing rather than constant combustion conditions were applied in many experiments, and a wide range of intermediate proportions were observed (cf. example in Table 1). The furan proportions are given relative to total $\mathrm{C}_{2}-\mathrm{C}_{8}$ hydrocarbons, determined from hydrocarbon analyses on the aluminium oxide column as previously described $[1,2]$. In smoke from glowing birchwood, the total amount of volatile furans may approach the total amount of volatile hydrocarbons, whereas the proportion of furans is only a few percent in smoke from flaming birchwood. The concentration of 2-methylfuran may be higher than that of furan from glowing, but the proportion of furan relative to $C_{5}-C_{7}$ furans increases with increasing combustion efficiency. The strikingly lowered proportions of the aromatic furans from flaming combustion contrast with an increased proportion of benzene, included for comparison as the major aromatic hydrocarbon. The proportions of specific hydrocarbons change in a characteristic way [2], and ethyne, ethene and benzene are major components from flaming wood burning. 
Table 2

Quantitative proportions in smoke from glowing and flaming combustion of birchwood ${ }^{\text {a }}$.

\begin{tabular}{lcc}
\hline & Glowing & Flaming \\
\hline Total $\mathrm{C}_{2}-\mathrm{C}_{8}$ hydrocarbons & 100 & 100 \\
Total $\mathrm{C}_{4}-\mathrm{C}_{7}$ furans & 60 & 3 \\
Furan & 20 & 2 \\
2-Methylfuran & 25 & 1 \\
Benzene & 5 & 10 \\
\hline
\end{tabular}

${ }^{a}$ The concentration of total $C_{2}-C_{8}$ hydrocarbons (index 100) is typically more than 10 times higher for flaming than for glowing combustion [2]. Proportions calculated mainly from gas chromatographic results obtained with the aluminium oxide column. 
The quantitative ratio between total furans and $\mathrm{CO}_{2}$ was in the range $0.1-1 \%$ for smoke from glowing and smouldering, and 2-4 orders of magnitude lower for samples from flaming combustion. The ratio between total $\mathrm{C}_{2}-\mathrm{C}_{8}$ hydrocarbons and $\mathrm{CO}_{2}$ decreased similarly but only by $1-2$ orders of magnitude [2]. In chimney samples from wood-stove burning, the proportions of furans, as compared with total $\mathrm{C}_{2}-\mathrm{C}_{8}$ hydrocarbons, were only about $1 \%$ and $10 \%$, respectively, for smoke from flaming fire and from glowing birchwood remainders. It is concluded that high emissions and concentrations of furans are to be expected only from glowing and smouldering combustion. Under these conditions, the proportions between different furans were found to be fairly uniform. The last column in Table 1 indicates typical quantitative ratios for the 14 most prominent recorded furans.

Comparisons with burning of other biomass samples indicated especially high proportions of furans from birchwood. This may partly be due to a content as high as about $30 \%$ of the polysaccharide xylan in hardwood from birch. Pyrolysis of wood polysaccharides gives rise to large proportions of anhydro sugars and other furan-related compounds [3,4,5,9], including the suspected carcinogen 2-furaldehyde [9]. These compounds may be precursors of the reported volatile furans. On the other hand, observed similar ratios between different furans from other biomass samples indicate that furans are useful as tracers of smoke from all kinds of inefficient biomass combustion. 


\section{References}

[1] G. Barrefors and G. Petersson, J. Chromatogr. A, 710 (1995) 71.

[2] G. Barrefors and G. Petersson, Chemosphere 30 (1995) 1551.

[3] L.M. McKenzie, W.M. Hao, G.N. Richards and D.E. Ward, Atmos. Environ. 28 (1994) 3285 .

[4] O. Faix, I. Fortmann, J. Bremer and D. Meier, Holz Roh - Werkst. 49 (1991) 213.

[5] O. Faix, I. Fortmann, J. Bremer and D. Meier, Holz Roh - Werkst. 49 (1991) 299.

[6] W. Baltes and G. Bochmann, Z. Lebensm, Unters. Forsch. 184 (1987) 179.

[7] T. Börjesson, U. Stöllman and J. Schnürer, Appl. Environ. Microbiol. 58 (1992) 2599.

[8] A.-L. Sunesson, W.H.J. Vaes, C.-A. Nilsson, G. Blomquist, B. Andersson and R. Carlsson, Appl. Environ. Microbiol. 61 (1995) 2911.

[9] L.M. McKenzie, W.M. Hao, G.N. Richards and D.E. Ward, Environ. Sci. Technol. 29 (1995) 2047. 